\title{
Mechanisms of Core Chinese Herbs against Colorectal Cancer: A Study Based on Data Mining and Network Pharmacology
}

\author{
Tong Lin $\mathbb{D},{ }^{1}$ Caijun Liang, ${ }^{1}$ Wenya Peng, ${ }^{1}$ Yuqin Qiu, ${ }^{1}$ and Lisheng Peng $\mathbb{D}^{2}$ \\ ${ }^{1}$ The Fourth Clinical Medical School, Guangzhou University of Chinese Medicine, Shenzhen 518033, China \\ ${ }^{2}$ Shenzhen Hospital of Traditional Chinese Medicine, Shenzhen 518033, China \\ Correspondence should be addressed to Lisheng Peng; lisheng_peng@outlook.com
}

Received 26 June 2020; Revised 26 September 2020; Accepted 10 October 2020; Published 28 October 2020

Academic Editor: Yu Hsiang Kuan

Copyright (c) 2020 Tong Lin et al. This is an open access article distributed under the Creative Commons Attribution License, which permits unrestricted use, distribution, and reproduction in any medium, provided the original work is properly cited.

Colorectal cancer (CRC) is now the second most deadly cancer globally. Chinese herbal medicine (CHM) plays an indispensable role in CRC treatment in China. However, the core herbs (the $\mathrm{CHs)} \mathrm{in} \mathrm{the} \mathrm{treatment} \mathrm{of} \mathrm{CRC} \mathrm{and} \mathrm{their} \mathrm{underlying} \mathrm{therapeutic}$ mechanisms remain unclear. This study aims to uncovering the $\mathrm{CHs}$ and their mechanisms of action of CRC treatment, applying data mining and network pharmacology approach. First, CHM prescriptions treating CRC were collected from clinical studies from the Chinese National Knowledge Infrastructure (CNKI) and MEDLINE databases, and the CHs were identified through data mining. Then, the bioactive compounds and the corresponding putative targets of the $\mathrm{CHs}$ were obtained from three traditional Chinese medicine (TCM) databases. CRC related targets were acquired from three disease databases; the overlapping targets between the $\mathrm{CHs}$ and CRC were identified as the therapeutic targets. Subsequently, functional enrichment analysis was performed to elucidate the mechanisms of the $\mathrm{CHs}$ on CRC. Moreover, networks were constructed to screen the major bioactive compounds and therapeutic targets. Finally, prognostic values of the major target genes were evaluated by survival analysis, and molecular docking simulation was performed to assess the binding affinity of key targets and major bioactive compounds. It came out that 10 the CHs from 113 prescriptions and 190 bioactive compounds with 118 therapeutic targets were identified. The therapeutic targets were mainly enriched in the biological progress of transcription, apoptosis, and response to cytokine. Various cancer-associated signaling pathways, including microRNAs, TNF, apoptosis, PI3K-Akt, and p53, were involved. Furthermore, 15 major bioactive compounds and five key target genes (VEGFA, CASP3, MYC, CYP1Y1, and NFKB1) with prognostic significance were identified. Additionally, most major bioactive compounds might bind firmly to the key target proteins. This study provided an overview of the anti-CRC mechanisms of the $\mathrm{CHs}$, which might refer to the regulation of apoptosis, transcription, and inflammation.

\section{Introduction}

Colorectal cancer (CRC), including colon and rectal cancer, is the third most frequently diagnosed cancer and the second leading cause of cancer-related deaths worldwide [1]. Furthermore, the rising incidence of CRC at younger ages $(<50$ years-old) is a merging trend [2]. Hereditary accounts for approximately $10-20 \%$ of all CRC patients, while $60-65 \%$ of CRC cases arise from modifiable risk factors, such as smoking, red and processed meat intake dietary, excessive alcohol intake, and obesity [3]. Currently, surgical resection is still the only curative treatment for CRC patients. Nevertheless, due to its occult onset, most CRC patients are diagnosed in advanced stages when surgery is unavailable
[4]. Another issue is that major complications occur on up to $15 \%$ patients, and postoperative recurrence and metastasis are quite common $[4,5]$. Neoadjuvant and adjuvant chemoradiotherapy is now generally accepted standard treatment for locally advanced CRC, with the aim of reducing recurrence and metastasis [6-8]. Disappointing, long-course chemoradiotherapy brings adverse reactions, such as myelosuppression, cumulative neuropathy, gastrointestinal tract reaction, and organs damage, which badly reduce the quality of life of the patients, and even leads to interruption of therapies [9]. Additionally, chemoradiotherapy is often confronted with treatment resistance [10].

Chinese herbal medicine (CHM) plays an indispensable role in integrative therapy of malignancies in China. It has 
been widely reported that CHM prescriptions could improve survival and quality of life of CRC patients through the following effects: (1) preventing tumorigenesis, suppressing tumor growth, and reducing metastasis and recurrence [11-14]; (2)increasing sensitivity and alleviating side effects of chemo- or radiotherapy; (3) relieving tumor-related symptoms or surgery complications, such as fatigue, pain, loss of appetite, diarrhea, nausea, and vomiting; and (4) improving immunity, lessening the damages induced by conventional treatments, and ameliorating bone marrow suppression [9, 15-18]. However, CHM prescriptions in the treatment of CRC differ a lot, due to varied educational backgrounds and personal clinical experiences of different traditional Chinese medicine (TCM) doctors. The underlying patterns or the core herbs (the $\mathrm{CHs)} \mathrm{of} \mathrm{the} \mathrm{pre-}$ scriptions and the bioactive compounds and therapeutic mechanisms of the $\mathrm{CHs}$ are still in veil.

The application of data mining and machine learning can help people better understand the patterns of herbs use from abundant clinical prescriptions [19]. CHM effects in a multicomponent, multitarget, and multipathway mode, which makes it unique superiority in treating complex diseases, but causes difficulty in clarifying mechanisms of action; meanwhile. Integrated systems biology, bioinformatics, and poly-pharmacology, network pharmacology provides an effective solution to uncover the synergistic effects and underlying mechanisms of multicomponent and multitarget agents through network analysis [20, 21]. Molecular docking is an in silico structure-based method to predict ligand-target interactions at a molecular level, which has been widely used in drug discovery [22].

In the present study, the $\mathrm{CHs}$ in the treatment of CRC were identified through data mining from prescriptions in clinical studies. Then, the bioactive compounds, the putative targets, and mechanisms of the CHs acting on CRC were investigated by network pharmacology approach. Finally, survival analysis and molecular docking simulation were performed to strengthen the results of network pharmacology analysis. A flowchart of this study is described in Figure 1.

\section{Materials and Methods}

2.1. Inclusion and Data Mining of CHM Prescriptions. Clinical CHM prescriptions in the treatment of CRC were collected from studies from the Chinese National Knowledge Infrastructure (CNKI) and MEDLINE databases. The literature search was conducted in topic, title, and abstract, using the following search terms: ("traditional Chinese medicine") OR ("Chinese herb"*) OR (decoction) OR (prescription) AND (colorectal cancer) AND (clinical); the timespan was set from database creation to 1st June 2020.

The inclusion criteria for studies were as follows: (1) the first diagnosis of patients which was CRC, (2) clinical studies about oral CHM prescriptions treating CRC, combined with (chemo)radiotherapy or not, and (3) the prescriptions should be effective. Effectiveness is defined as statistically significant protective effects in the CHM treatment group, compared with the control group. The protective effects included but are not limited to the alleviation of cancerrelated symptoms and/or adverse reactions of (chemo)radiotherapy, prolongation of survival, improvement of quality of life, and immunity. The exclusion criteria were as follows: (1) review articles and laboratory experimental studies, (2) the constituent herbs in prescriptions not published in full, (3) studies in which the patients were treated with extract (s) of a single herb or Chinese patent medicine, and (4) the number of patients in any single group being less than 20 .

The CHM prescriptions were extracted from the eligible studies, and a database was formed. Then, frequency analysis was performed with SPSS software (version 21.0, IBM Corp., Armonk, NY, USA). And association rule analysis was conducted by a priori algorithm with SPSS Clementine software (version 12.0, SPSS Inc., Chicago, IL, USA), to identify the CHs from all prescriptions. In this study, association rules were set under the condition of support degree $\geq 20 \%$ and confidence degree $\geq 50 \%$.

\subsection{Bioactive Compounds and Putative Target of the CHs.} The compounds of all the CHs were collected from Traditional Chinese Medicine Systems Pharmacology Database and Analysis Platform (TCMSP, http://tcmspw.com/tcmsp. php) [23], Integrative Pharmacology-based Research Platform of TCM (TCMIP, http://www.tcmip.cn/) [24], and Bioinformatics Analysis Tool for Molecular Mechanisms of Traditional Chinese Medicine Database (BATMAN-TCM, http://bionet.ncpsb.org/batman-tcm/) [25].

Based on the TCMSP database, oral bioavailability $(\mathrm{OB}) \geq 30 \%$ and drug-likeness $(\mathrm{DL}) \geq 0.18$ were set as the screening criteria of bioactive compounds, which were labeled as "Mol ID" in the TCMSP database in the further study [26]. The putative targets corresponding to the bioactive compounds were collected from the above three TCM databases, and all target names were converted into gene symbols using the UniProt database (https://www.uniprot. org/) for the subsequent study.

2.3. Therapeutic Targets of the CHs in the Treatment of CRC. CRC related targets were obtained from the MalaCards (https://www.malacards.org/) [27], DisGeNET (http://www. disgenet.org/) [28] and the Comparative Toxicogenomics Database (CTD, http://ctdbase.org) [29], and the search terms used were "colorectal carcinoma" or "colorectal cancer." The overlapping targets between the CHs and CRC were determined using Venn diagram with an online tool named Venny 2.1 (https://bioinfogp.cnb.csic.es/tools/ venny/index.html). And the overlapping targets were recognized as the potential therapeutic targets of the $\mathrm{CHs}$ in CRC.

2.4. Functional Enrichment Analysis. To elucidate the therapeutic mechanisms of the $\mathrm{CHs}$ on $\mathrm{CRC}$, gene ontology (GO) functional enrichment analysis and Kyoto Encyclopedia of Genes and Genomes (KEGG) pathway enrichment analysis of therapeutic targets were performed using the 


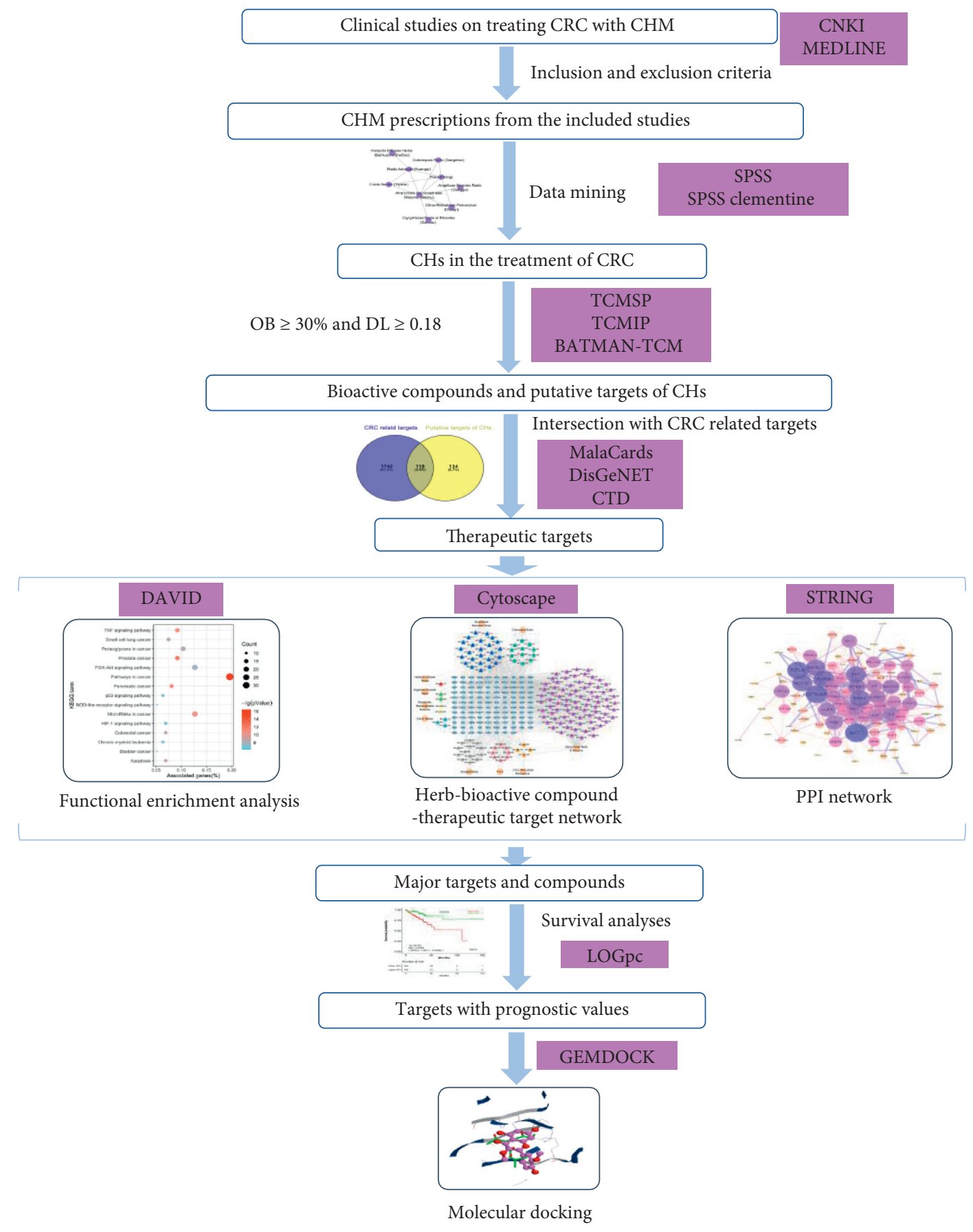

Figure 1: A flowchart of this study.

Database for Annotation, Visualization, and Integrated Discovery database (David, https://david.ncifcrf.gov/) [30]. The results with $P$ value $<0.05$ and false discovery rate $($ FDR $)<0.05$ were considered significant, which were visualized using the GraphPad Prism software (version 6.0, San Diego, CA, USA) and "ggplot2" package in R.

2.5. Network Construction, Major Compounds, and Major Targets Screening. Two networks were constructed in this study. (1) A herb-bioactive compound-therapeutic target network was established to show the interactions among the
CHs, bioactive compounds, and therapeutic targets. (2) A protein-protein interaction (PPI) network was built to explore the interactions among therapeutic targets. The PPI data of therapeutic targets were analyzed using Search Tool for the Retrieval of Interacting Genes/Proteins platform (STRING, https://string-db.org/) [31]. The species was set as "Homo sapiens", and the minimum confidence score was set as 0.9 .

The diagrammatic networks were constructed using Cytoscape software version 3.7.2 [32], and the major bioactive compounds and targets were screened by network 
topological parameters calculated with Network Analyzer plug-in. The topological parameter of degree value was used to describe the importance of a node in a network, which is defined as the number of edges linked to a certain node. A major node must have a degree value two times greater than the median degree value of all nodes in the network [33].

2.6. Survival Analysis of the Major Target Genes. The prognostic value of major target genes was assessed by survival analysis using a web server named Long-Term Outcome and Gene Expression Profiling Database of Pan-Cancers (LOGpc, http://bioinfo.henu.edu.cn/DatabaseList.jsp), which provides 13 types of survival terms for 28,098 patients from 26 malignancies [34]. All cases were categorized into high and low expression groups by the median expression of a certain therapeutic target gene; then overall survival (OS) and disease free survival (DFS) were analyzed by log-rank test. Kaplan-Meier (KM) curves, hazard ratio (HR), 95\% confidence intervals (CI), and log-rank $P$ value were generated online. The results with $P<0.05$ were considered statistically significant, and the targets correlated with patients' survivals were considered as the key targets.

2.7. Molecular Docking Simulation. To evaluate the binding potential of the major bioactive compounds of the $\mathrm{CH}$ with the key target proteins, molecular docking simulation was performed using GEMDOCK software (version 2.1, National Chiao Tung University, Hsinchu, Taiwan) [35]. The empirical scoring function of GEMDOCK is as follows: Fitness = van der Waal energy + hydrogen bonding energy+electro statistic energy. A fitness value was used to estimate the binding affinity of a protein and a ligand and the fitness value of the corresponding protein-original ligand complex was used as a comparison. The generic evolutionary method parameters were set as population size $=200$, generations $=70$, and number of solutions $=2$. All $3 \mathrm{D}$ crystal structures of target protein-original ligand complexes were downloaded as “.pdb” files from Protein Data Bank (PDB, http://www.rcsb.org/pdb/) [36]. All 3D molecular structures of original ligands and major bioactive compounds were downloaded as ".mol2" files from TCMSP database or ZINC database (http://zinc.docking.org) [37].

\section{Results}

3.1. Results of Data Mining. 1130 and 131 studies were obtained from CNKI and MEDLINE databases, respectively. According to the inclusion and exclusion criteria above, 108 and 3 clinical studies were included from CNKI (published in Chinese) and MEDLINE (published in English) databases, respectively. A list of the clinical studies included in this study is provided in Supplementary Table 1 . Then, a total of $113 \mathrm{CHM}$ prescriptions treating CRC were extracted from the 111 clinical studies. There were 196 different Chinese herbs used in all prescriptions, and the frequency analysis of herbs revealed that the ten most frequently used Chinese herbs were Atractylodis Macrocephalae Rhizoma (Bai-Zhu), Poria (Fu-Ling), Radix Astragali (Huang-Qi), Glycyrrhizae Radix et Rhizoma (Gan-Cao), Codonopsis Radix (Dang-Shen), Coicis Semen (Yi-Yi-Ren), Citrus Reticulatae Pericarpium (Chen-Pi), Hedyotis Diffusae Herba (Bai-Hua-She-She-Cao), Angelicae Sinensis Radix (Dang-Gui), and Scutellariae Barbatae Herba (Ban-Zhi-Lian), which were recognized as the $\mathrm{CHs}$ in the treatment of CRC. The usage frequency of each $\mathrm{CH}$ is shown in the second and third columns of Table 1. Besides, the association rule analysis revealed that nine herbs out of the $\mathrm{CHs}$ were often used together, whose associations are shown in Figure 2(a).

3.2. Bioactive Compounds and Putative Therapeutic Targets of the CHs. The eligible bioactive compounds of every herb were merged from three TCM databases, bioactive compounds reiterated, or without putative targets were removed. Totally, 190 bioactive compounds and 252 putative targets of the $\mathrm{CHs}$ were included. The final counts of bioactive compounds of each $\mathrm{CH}$ are shown in the fourth column of Table 1, and duplicates existed among different the $\mathrm{CHs}$.

In terms of CRC related targets, 838 targets were obtained from MalaCards, and the top 1000 targets were collected from DisGeNET and CTD databases, according to their rank of inference score, respectively. A total of 1860 CRC related targets were found after removing the duplicates. Subsequently, 118 overlapping targets between the $\mathrm{CHs}$ and $\mathrm{CRC}$ were identified using the Venn diagram (Figure 2(b)), which were considered as the potential therapeutic targets of the CHs for CRC.

3.3. GO and KEGG Pathway Enrichment Analysis. Functional enrichment analysis was performed for 118 therapeutic targets. In total, 17 GO terms were identified, including 8 biological process (BP) terms, 6 molecule function (MF) terms, and 3 cellular component (CC) terms $(P$ value and FDR both $<0.05)$. For BP, the targets mainly participated in the regulation of transcription, apoptosis, and response to cytokine. For MF, the targets were mainly responsible for the activity of steroid hormone receptor and transcription factor, as well as binding of chromatin, DNA, and steroid. For CC, the targets were mainly distributed in nucleus, extracellular space, and cytoplasm. The five most significantly enriched GO terms are shown as bar plots in Figure 3(a).

Besides, 44 KEGG pathway terms were enriched, many of which were pathways of specific kinds of cancer. Moreover, microRNAs in cancer and tumor necrosis factor (TNF), apoptosis, PI3K-Akt, and p53 signaling pathways, and so on were enriched. The top fifteen most significantly enriched KEGG terms are shown as a bubble chart in Figure 3(b). 
TABLE 1: Usage frequency and counts of bioactive compounds of the CHs.

\begin{tabular}{lccc}
\hline Core herbs & Frequency & Proportion of all prescriptions & Counts of bioactive compounds \\
\hline Atractylodis macrocephalae rhizoma & 98 & 0.87 & 4 \\
Poria & 80 & 0.71 & 7 \\
Astragali radix & 75 & 0.66 & 17 \\
Glycyrrhizae radix et rhizoma & 70 & 0.62 & 91 \\
Codonopsis radix & 63 & 0.56 & 18 \\
Coicis semen & 50 & 0.44 & 6 \\
Citrus reticulatae pericarpium & 46 & 0.41 & 6 \\
Hedyotis diffusae herba & 44 & 0.39 & 5 \\
Angelicae sinensis radix & 39 & 0.35 & 3 \\
Scutellariae barbatae herba & 34 & 0.30 & 30 \\
\hline
\end{tabular}

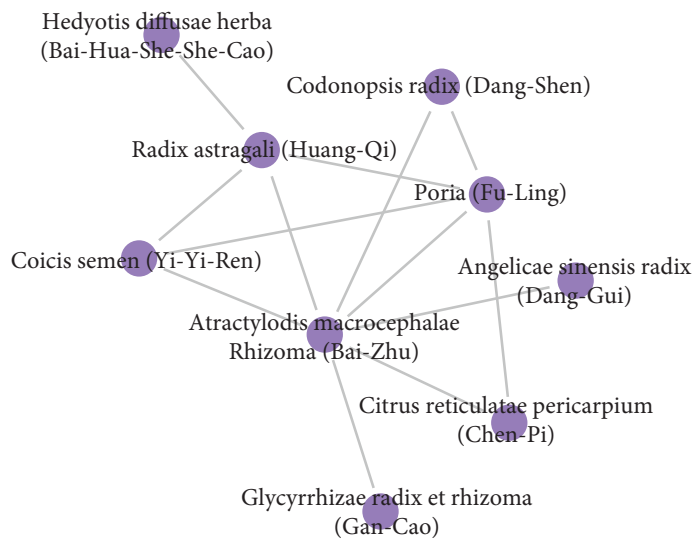

(a)

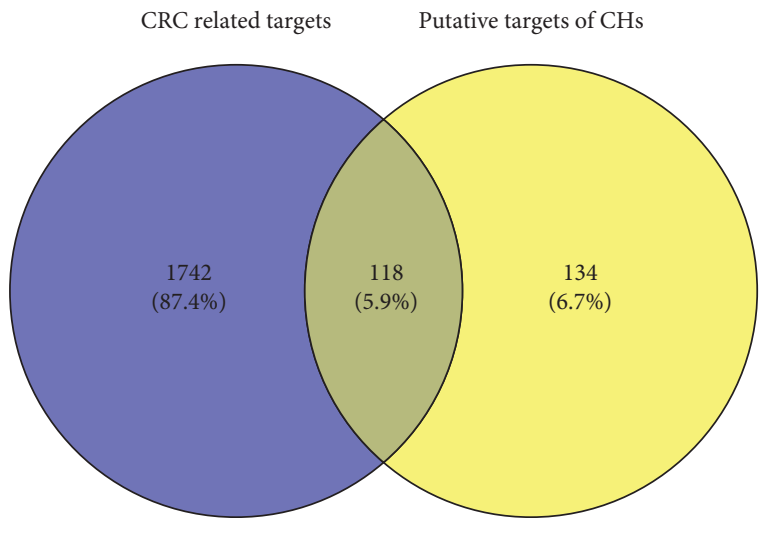

(b)

Figure 2: The association rules network of the $\mathrm{CH}$ and the Venn diagram; (a) the association rules network of the CHs. An edge represents an association rule between two herbs, which met the criteria of support degree $\geq 20 \%$ and confidence degree $\geq 50 \%$, and (b) the Venn diagram showing overlapped targets between the $\mathrm{CHs}$ and CRC.

\subsection{The Network of Herb-Bioactive Compound-Therapeutic} Target. The network of herb-bioactive compound-therapeutic target was composed of 277 nodes (10 herbs, 149 bioactive compounds, and 118 targets) and 1240 edges (Figure 3). Some bioactive compounds were absent in the network, in default of interaction with any therapeutic target. Among 149 compounds, quercetin might act on the most targets (degree $=59$ ), followed by wogonin $($ degree $=33)$ and luteolin (degree $=25)$, the cumulative number of targets of the above three compounds was 82 (over $55 \%$ of all therapeutic targets). The information of the top 15 bioactive compounds with the most interactive therapeutic targets is shown in Table 2.

3.5. PPI Network and Major Targets Screening. Based on the PPI analysis from the STRING, 12 targets were not involved in protein interactions; thus, the final PPI network consisted of 106 nodes (targets) and 364 edges (Figure 4). The median degree value of the PPI network was five, and 25 targets met the criteria of major targets in this network. The major targets interacted extensively with other proteins, suggesting their crucial roles in the development and progression of CRC. Table 3 lists the detailed information on these major targets.

3.6. Prognostic Significance of Major Target Genes. Survival analysis was performed for every major target gene using the LOGpc server. The results showed that none of the major target genes showed significant association with OS in CRC patients. Nevertheless, high expression of VEGFA was associated with shorter DFS; high expression of vascular endothelial growth factor A (VEGFA), caspase 3 (CASP3), Myc protooncogene protein (MYC), cytochrome P450 enzymes 1A1 (CYP1A1), and NF- $\kappa$ B p105/p50 subunit (NFKB1) were associated with longer DFS in CRC patients. So, these five targets were chosen as the key targets of the $\mathrm{CH}$ treating CRC; the $\mathrm{KM}$ survival curves of them are presented in Figure 5. And they were selected to perform molecular docking simulation.

3.7. Results of Molecular Docking. Because the NFKB1 protein-original ligand complex was unavailable from the 


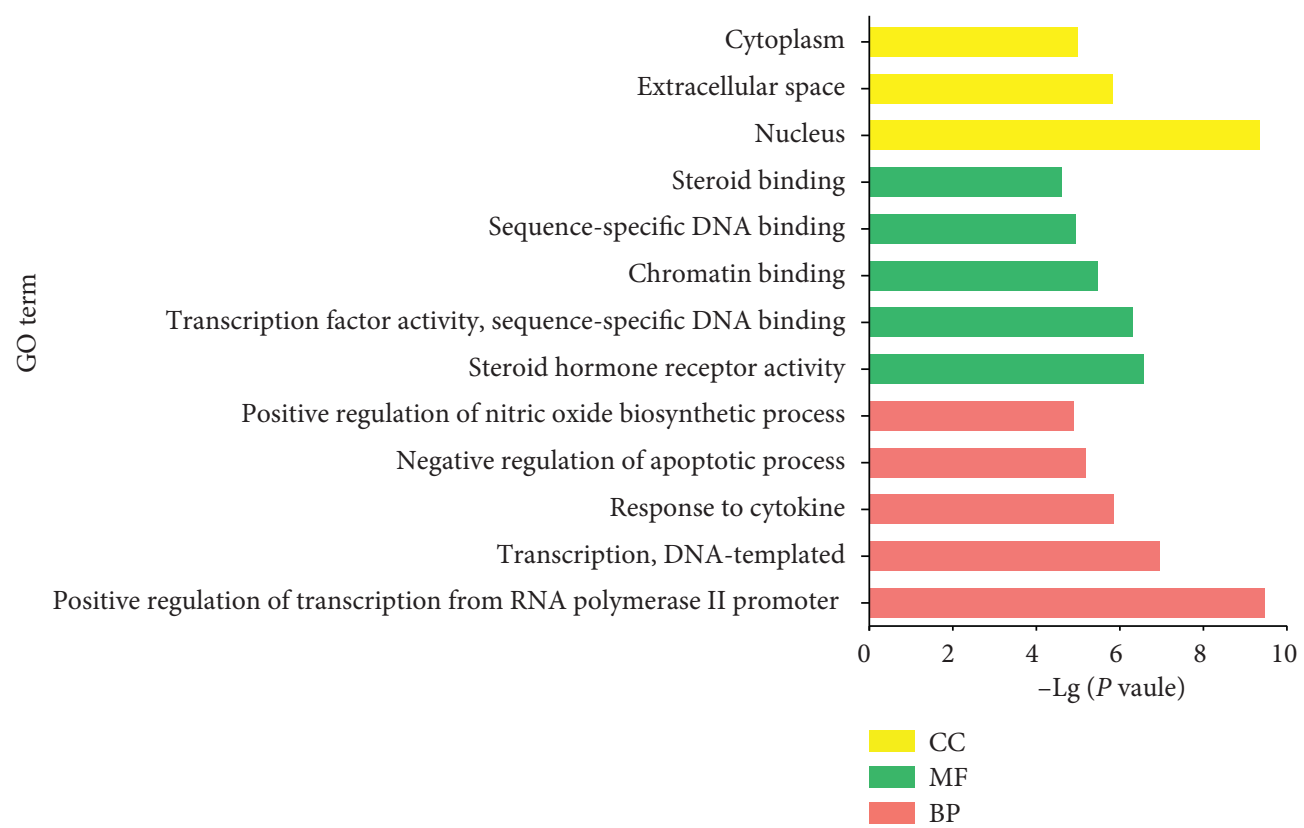

(a)

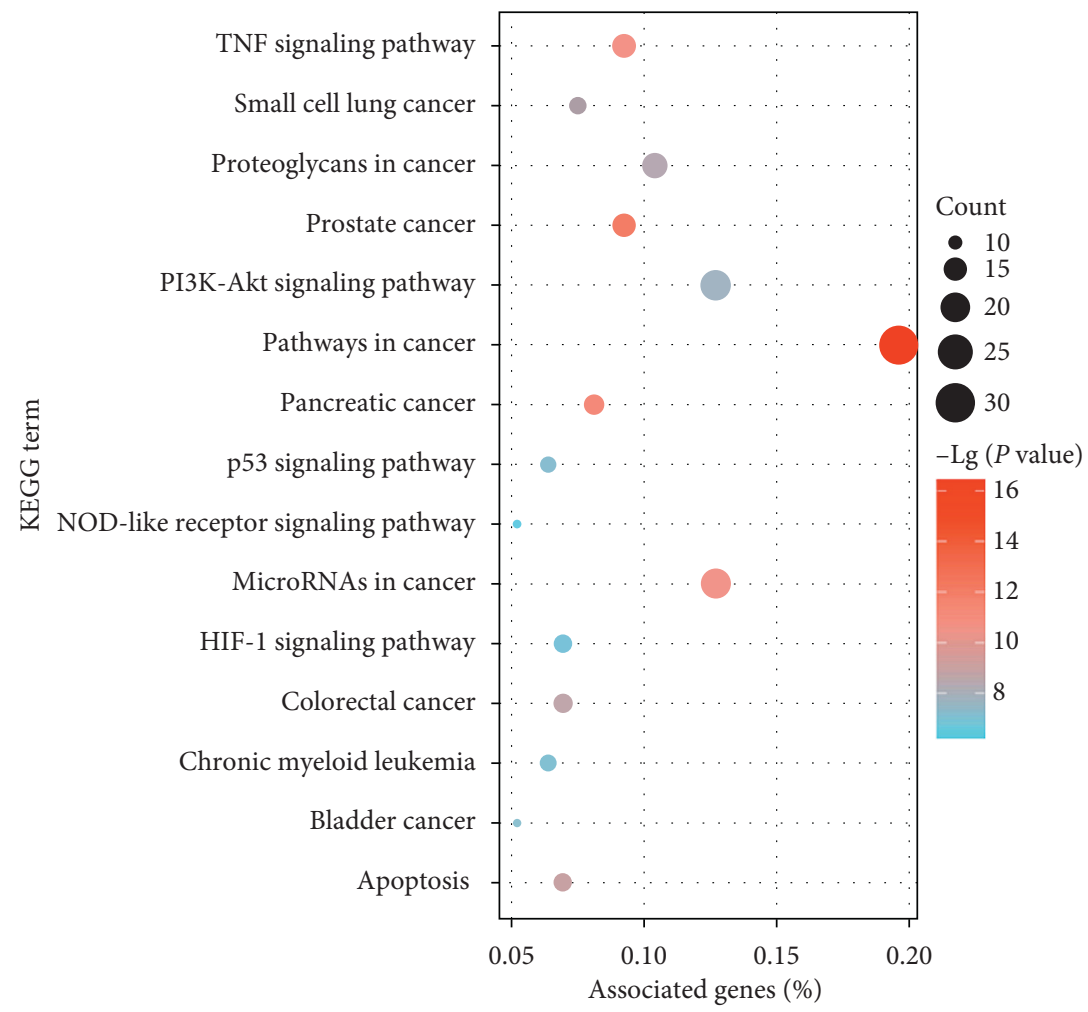

(b)

FIGURE 3: Functional enrichment analysis of therapeutic targets of the CHs for CRC. (a) The five most significantly enriched BP and MF terms. For CC, only three terms were significantly enriched. (b) The top 15 most significantly enriched KEGG terms. The bubble size represents the count of therapeutic targets enriched in a certain pathway; the abscissa shows the percentage of target genes to the background genes of a certain pathway.

PDB database, four key target proteins, VEGFA, CASP3, MYC, and CYP1Y1, were docked with 15 bioactive compound ligands. Because a lower fitness value signifies a stabler binding, the results of molecular docking implied four key targets might bind firmer with the 15 bioactive compounds, compared with their corresponding original 
TABLE 2: Information of the top 15 bioactive compounds of the $\mathrm{CHs}$.

\begin{tabular}{|c|c|c|c|c|c|}
\hline Mol ID & Compound name & OB & $\mathrm{DL}$ & $\begin{array}{c}\text { Count of } \\
\text { therapeutic } \\
\text { targets }\end{array}$ & Source of the CHs \\
\hline MOL000098 & Quercetin & 46.43 & 0.28 & 59 & $\begin{array}{c}\text { Astragali radix, Hedyotis diffusae herba, scutellariae barbatae } \\
\text { herba, glycyrrhizae radix et rhizoma }\end{array}$ \\
\hline MOL000173 & Wogonin & 30.68 & 0.23 & 33 & Scutellariae barbatae herba \\
\hline MOL000006 & Luteolin & 36.16 & 0.25 & 25 & Scutellariae barbatae Herba, Codonopsis radix \\
\hline MOL000287 & Eburicoic acid & 38.7 & 0.81 & 25 & Poria \\
\hline MOL000422 & Kaempferol & 41.88 & 0.24 & 25 & Astragali Radix, glycyrrhizae radix et rhizoma \\
\hline MOL002933 & $\begin{array}{l}5,7,4^{\prime} \text {-Trihydroxy-8- } \\
\text { methoxyflavone }\end{array}$ & 36.56 & 0.27 & 24 & Scutellariae barbatae herba \\
\hline MOL004961 & Quercetin der. & 46.45 & 0.33 & 23 & Glycyrrhizae radix et rhizoma \\
\hline MOL010586 & Formononetin & 66.39 & 0.21 & 23 & Glycyrrhizae radix et rhizoma \\
\hline MOL000239 & Jaranol & 50.83 & 0.29 & 20 & Astragali Radix, glycyrrhizae radix et rhizoma \\
\hline MOL000280 & Dehydrotumulosic acid & 31.07 & 0.82 & 20 & Poria \\
\hline MOL000289 & Pachymic acid & 33.63 & 0.81 & 19 & Poria \\
\hline MOL002910 & Carthamidin & 41.15 & 0.24 & 19 & Scutellariae barbatae herba \\
\hline MOL002844 & Pinocembrin & 64.72 & 0.18 & 18 & Glycyrrhizae radix et rhizoma \\
\hline MOL002714 & Baicalein & 33.52 & 0.21 & 16 & Scutellariae barbatae herba \\
\hline MOL004355 & Spinasterol & 42.98 & 0.76 & 16 & Codonopsis radix \\
\hline
\end{tabular}

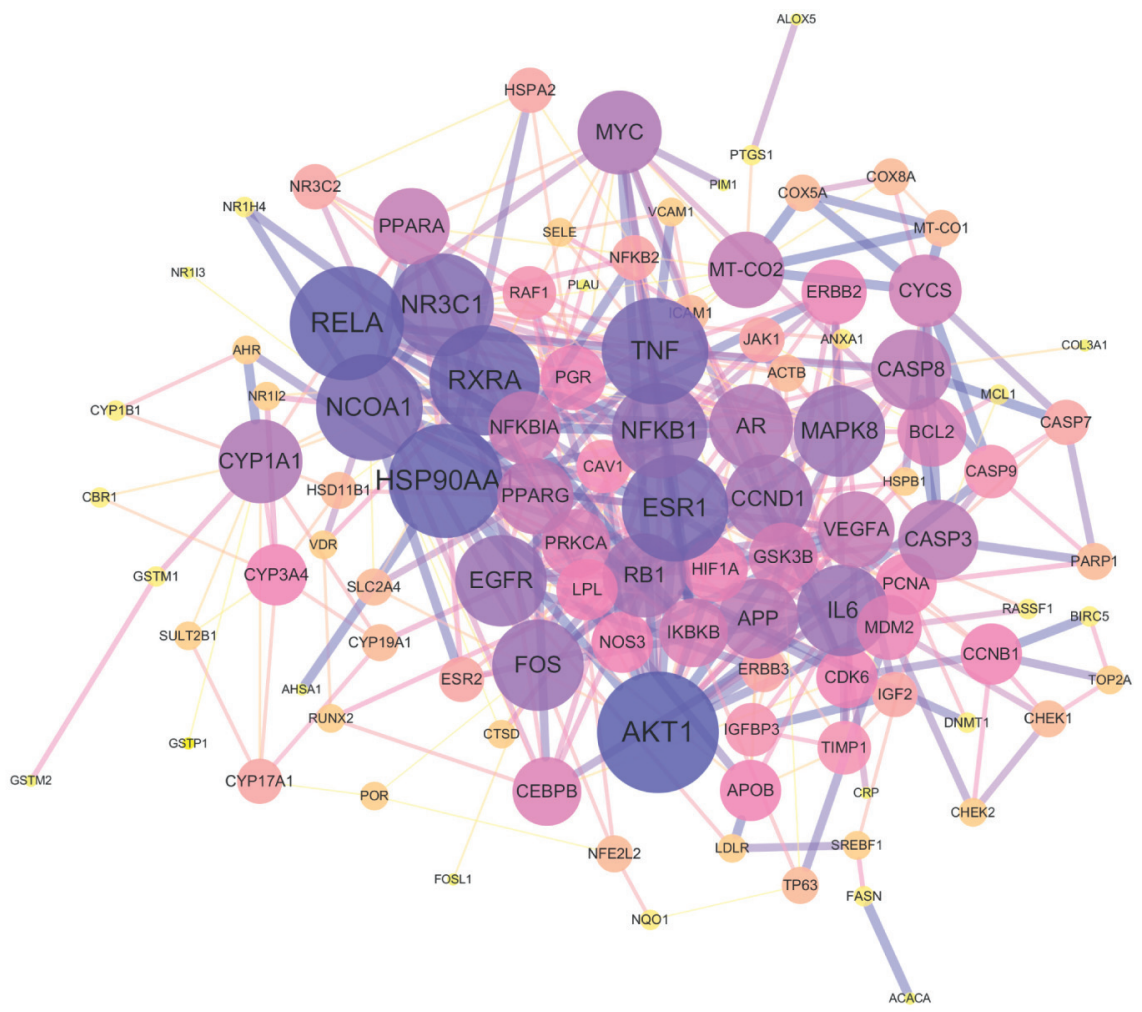

Figure 4: The PPI network of the therapeutic targets. The size and the color of nodes are proportional to their degree values; small size and bright color represent low degree values. The thickness and the color of edges are proportional to the interaction levels between nodes.

ligands (Table 4). Furthermore, it is easy to find out that CYP1A1 protein has the best binding affinities with the 15 compound ligands. Figure 6 shows the docking model of each target protein and the bioactive compound with the firmest binding.

\section{Discussion}

The current study could be divided into two major parts, which realized the inference from clinical experience to molecular mechanisms. In the first part, we identified the effective $\mathrm{CHs}$ in the treatment of CRC from abundant 
TABle 3: The major targets of the CHs in the treatment of CRC.

\begin{tabular}{|c|c|c|c|}
\hline No. & Target name & Gene symbol & Degree value in the PPI network \\
\hline 1 & RAC-alpha serine/threonine-protein kinase & Akt1 & 23 \\
\hline 2 & Heat shock protein HSP 90 -alpha & HSP90AA1 & 21 \\
\hline 3 & Transcription factor p65 & RELA & 21 \\
\hline 4 & Retinoic acid receptor RXR-alpha & RXRA & 20 \\
\hline 5 & Tumor necrosis factor & TNF & 19 \\
\hline 6 & Nuclear receptor coactivator 1 & NCOA1 & 19 \\
\hline 7 & Estrogen receptor & ESR1 & 19 \\
\hline 8 & Glucocorticoid receptor & $\mathrm{NR} 3 \mathrm{C} 1$ & 18 \\
\hline 9 & Nuclear factor NF-kappa-B p105 subunit & NFKB1 & 16 \\
\hline 10 & Epidermal growth factor receptor & EGFR & 15 \\
\hline 11 & Mitogen-activated protein kinase 8 & MAPK8 & 15 \\
\hline 12 & Proto-oncogene c-Fos & FOS & 15 \\
\hline 13 & Interleukin-6 & IL6 & 15 \\
\hline 14 & G1/S-specific cyclin-D1 & CCND1 & 14 \\
\hline 15 & Cytochrome P450 1A1 & CYP1A1 & 13 \\
\hline 16 & Retinoblastoma-associated protein & $\mathrm{RB1}$ & 13 \\
\hline 17 & Myc protooncogene protein & MYC & 13 \\
\hline 18 & Androgen receptor & AR & 13 \\
\hline 19 & Caspase- 8 & CASP8 & 12 \\
\hline 20 & Caspase-3 & CASP3 & 12 \\
\hline 21 & Amyloid beta A4 protein & APP & 12 \\
\hline 22 & Cytochrome $\mathrm{c}$ oxidase subunit 2 & MT-CO2 & 11 \\
\hline 23 & Peroxisome proliferator-activated receptor gamma & PPARG & 11 \\
\hline 24 & Vascular endothelial growth factor A & VEGFA & 11 \\
\hline 25 & Peroxisome proliferator-activated receptor alpha & PPARA & 11 \\
\hline
\end{tabular}
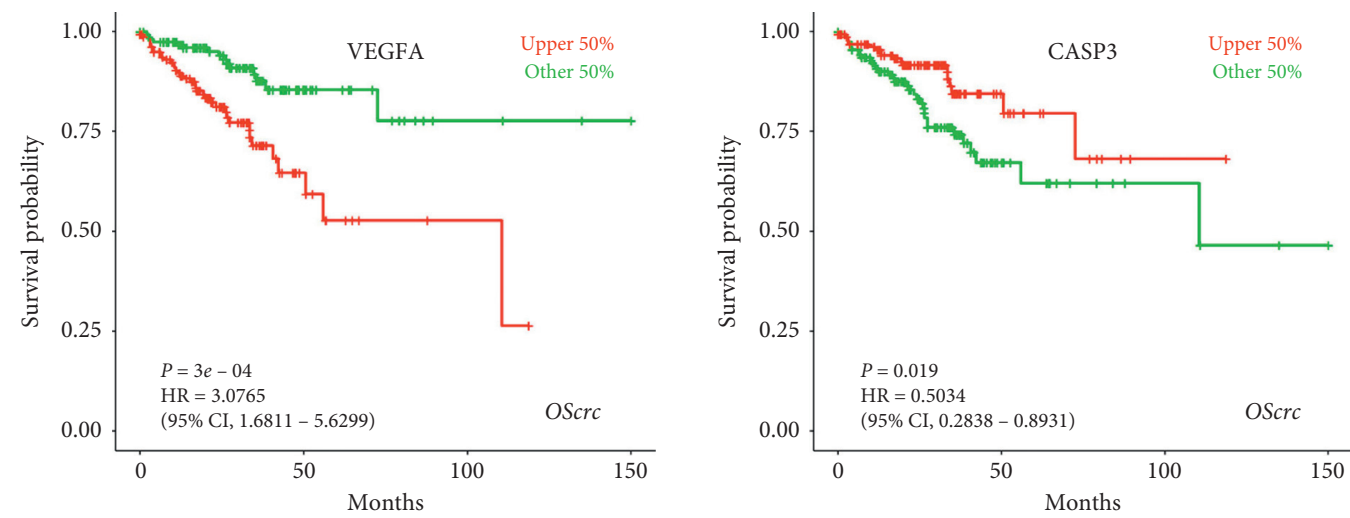

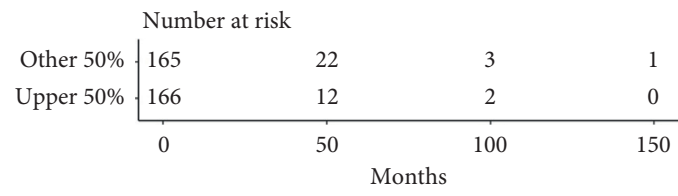

(a)

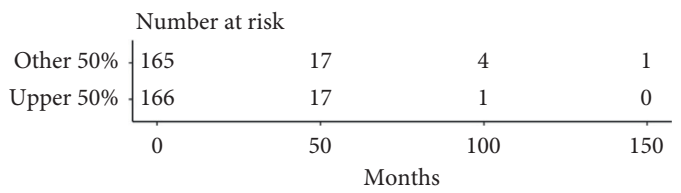

(b)

Figure 5: Continued. 


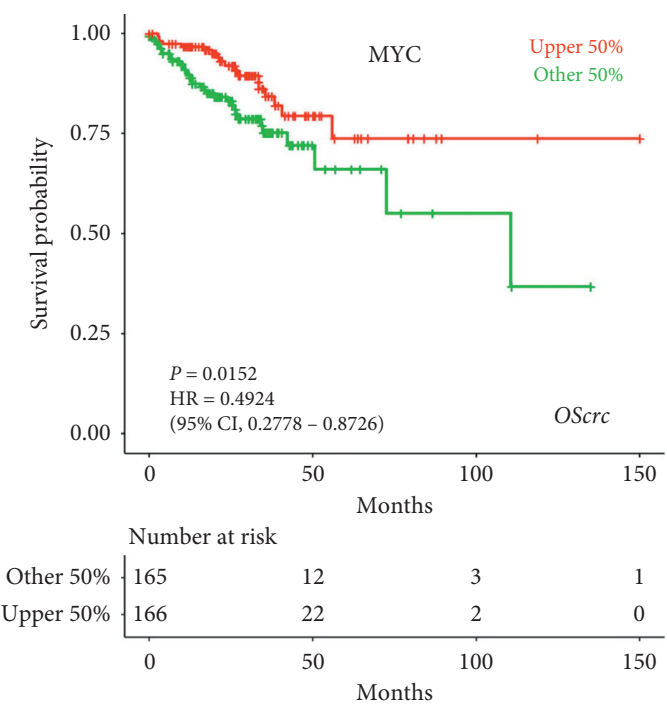

(c)
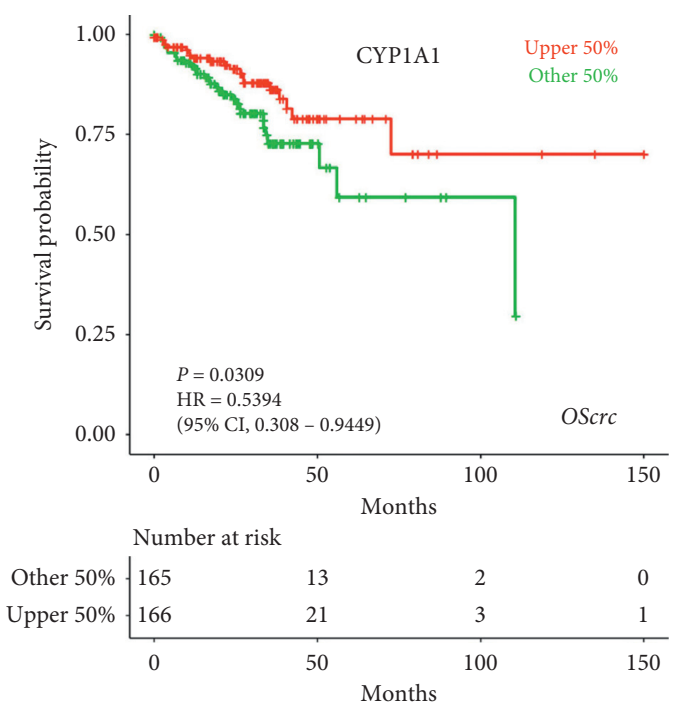

(d)

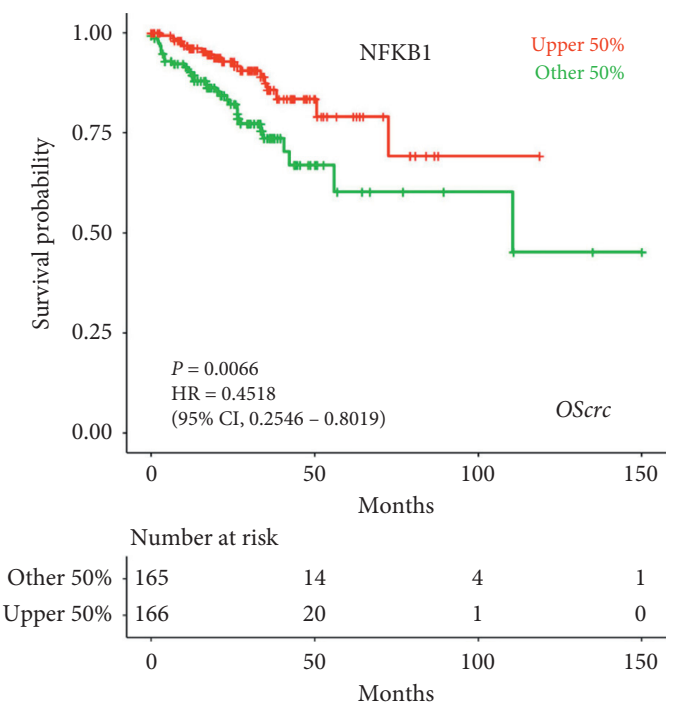

(e)

FIGURE 5: Five key target genes with prognostic significance for DFS in CRC patients. KM survival curves for (a) VEGFA, (b) CASP3, (c) MYC, (d) CYP1Y1, and (e) NFKB1.

TABLe 4: The results of molecular docking simulation.

\begin{tabular}{|c|c|c|c|c|c|}
\hline Target protein (PDB ID) & Ligand & Fitness $(\mathrm{kcal} / \mathrm{mol})$ & Target protein (PDB ID) & Ligand & Fitness $(\mathrm{kcal} / \mathrm{mol})$ \\
\hline \multirow{16}{*}{ CASP3 (3GJR) } & MOL004961 & -120.03 & \multirow{16}{*}{ MYC (5i4z) } & MOL004355 & -88.88 \\
\hline & MOL000098 & -110.49 & & MOL000287 & -88.70 \\
\hline & MOL000422 & -110.21 & & MOL004961 & -88.20 \\
\hline & MOL000006 & -109.54 & & MOL000239 & -85.61 \\
\hline & MOL002714 & -107.83 & & MOL000098 & -82.74 \\
\hline & MOL000289 & -104.90 & & MOL000289 & -82.22 \\
\hline & MOL000280 & -101.64 & & MOL002933 & -80.59 \\
\hline & MOL000239 & -100.51 & & MOL000173 & -80.46 \\
\hline & MOL000287 & -99.34 & & MOL000280 & -79.61 \\
\hline & MOL000173 & -97.94 & & MOL000006 & -79.57 \\
\hline & MOL002844 & -97.52 & & MOL002714 & -79.00 \\
\hline & MOL002933 & -96.42 & & MOL000422 & -77.54 \\
\hline & MOL002910 & -96.29 & & MOL002910 & -77.38 \\
\hline & MOL010586 & -88.88 & & MOL010586 & -76.89 \\
\hline & MOL004355 & -87.31 & & MOL002844 & -73.26 \\
\hline & DZE* & -66.14 & & $\mathrm{GOL}^{*}$ & -44.23 \\
\hline
\end{tabular}


TABle 4: Continued.

\begin{tabular}{|c|c|c|c|c|c|}
\hline Target protein (PDB ID) & Ligand & Fitness $(\mathrm{kcal} / \mathrm{mol})$ & Target protein (PDB ID) & Ligand & Fitness $(\mathrm{kcal} / \mathrm{mol})$ \\
\hline \multirow{16}{*}{ CYP1A1 (6dwm) } & MOL000239 & -158.75 & \multirow{16}{*}{ VEGFA (6d3o) } & MOL000289 & -102.14 \\
\hline & MOL000280 & -156.15 & & MOL000280 & -92.96 \\
\hline & MOL004961 & -153.75 & & MOL000098 & -91.14 \\
\hline & MOL002933 & -151.42 & & MOL002910 & -91.00 \\
\hline & MOL000006 & -150.57 & & MOL000006 & -90.53 \\
\hline & MOL000098 & -147.94 & & MOL004961 & -90.53 \\
\hline & MOL000422 & -146.91 & & MOL000239 & -82.89 \\
\hline & MOL002714 & -144.12 & & MOL004355 & -82.03 \\
\hline & MOL000287 & -141.47 & & MOL002844 & -81.16 \\
\hline & MOL004355 & -140.61 & & MOL002933 & -80.73 \\
\hline & MOL000173 & -137.33 & & MOL000287 & -80.18 \\
\hline & MOL000289 & -135.84 & & MOL000422 & -80.08 \\
\hline & MOL010586 & -133.70 & & MOL002714 & -77.53 \\
\hline & MOL002910 & -119.95 & & MOL000173 & -76.44 \\
\hline & MOL002844 & -114.64 & & MOL010586 & -70.71 \\
\hline & CPS* & -107.82 & & NLE $^{*}$ & -69.12 \\
\hline
\end{tabular}

${ }^{*}$ DZE, CPS, GOL, and NLE are the names of the original ligands in the PDB database.

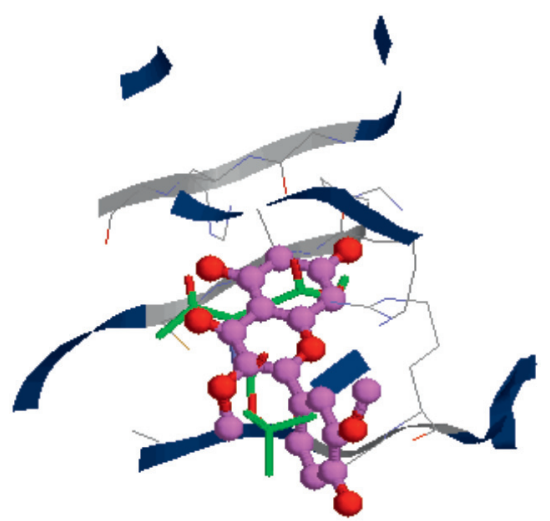

(a)

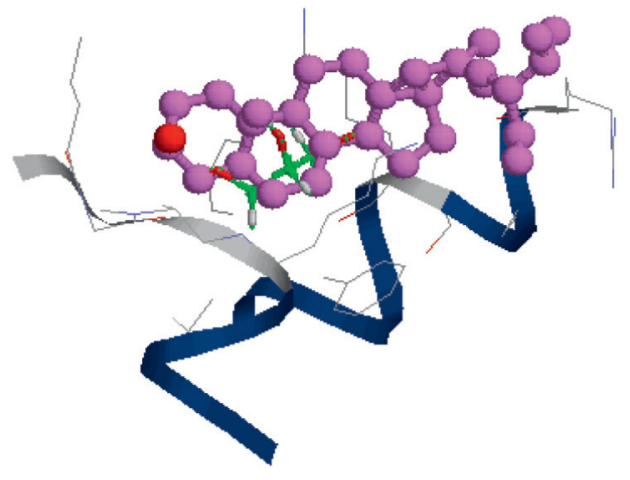

(c)

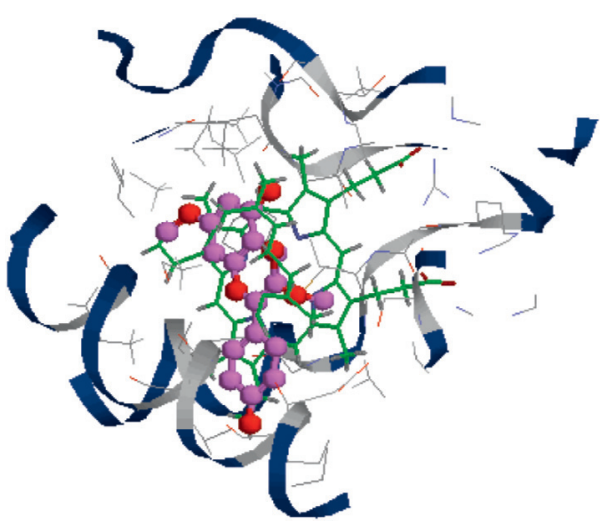

(b)

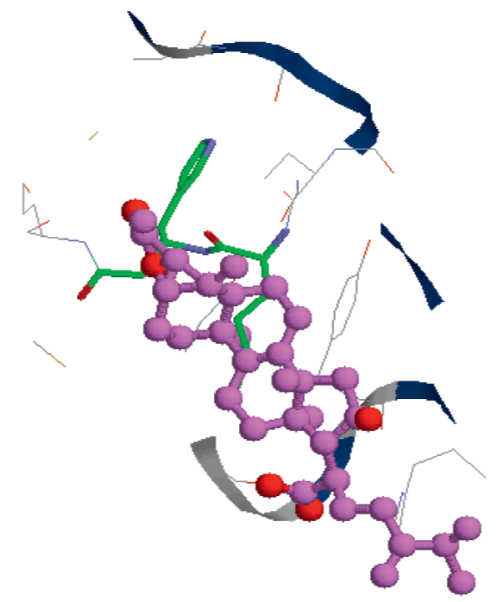

(d)

FIGURE 6: Molecular docking models of target proteins with bioactive compounds. (a) CASP3-MOL004961, (b) CYP1A1-MOL000239, (c) MYC-MOL004355, and (d) VEGFA-MOL000289. The original ligands are labeled on green color, and the predicted poses of bioactive compounds are labeled on pink color. 
CHM prescriptions through data mining. In the second part, we deduced the therapeutic targets and mechanisms of the $\mathrm{CHs}$ acting on CRC by network pharmacology approach.

Firstly, ten Chinese herbs were identified as the $\mathrm{CH}$ s in the treatment of CRC, according to their high frequency of use and association rules with each other (Table 1 and Figure 2(a)). In CHM theory, Atractylodis Macrocephalae Rhizoma, Radix Astragali, Glycyrrhizae Radix et Rhizoma, Codonopsis Radix, and Angelicae Sinensis Radix are classified as tonic herbs to replenish the body's Qi (akin to the body's healthy energy) and blood. Poria, Coicis Semen, and Citrus Reticulatae Pericarpium are classified as dampness-dispelling herbs. Hedyotis Diffusae Herba and Scutellariae Barbatae Herba are classified as heat-clearing and detoxifying herbs. In the TCM theory of pathophysiology, deficiency of Qi and excess of toxic heat are the two most internal causes of CRC. What is more, deficiency of Qi usually leads to extra dampness, which is similar to the accumulation of metabolites in the body. Thus, the effects of the $\mathrm{CHs}$ were accorded with the TCM pathogenesis of CRC. Congruously, previous studies had demonstrated the benefits of the most $\mathrm{CHs}$ on CRC patients, including inhibiting side effects of chemotherapy and improving tumor response [38-41].

Then, the bioactive compounds and putative targets of the $\mathrm{CHs}$ were collected. The overlapping targets between the $\mathrm{CHs}$ and $\mathrm{CRC}$ were considered as the potential therapeutic targets of $\mathrm{CHs}$ for CRC (Figure 2(b)). The functional enrichment analysis uncovered the therapeutic targets might mainly participate in the regulation of transcription, binding of DNA, reacting to inflammation, and regulation of apoptosis (Figure 3(a)). And the involved signaling pathways were mostly cancer-related, including microRNAs, TNF, apoptosis, PI3K-Akt, and p53 pathways (Figure 3(b)).

MicroRNAs (miRNAs) participate in tumorigenesis, progression, invasion, and drug resistance in cancers, including CRC. The activation of the PI3K/Akt pathway in cancer promotion had been widely learned, and miRNAs can affect CRC by regulating PI3K/Akt pathway in dual ways. For example, miRNA21 and miRNA200c could promote CRC by downregulating phosphatase and tensin homolog (PTEN), a negative regulator of PI3K, while miRNA106a and miRNA1 could suppress CRC by activating PTEN or directly blocking PI3K/Akt pathway $[42,43]$. Chronic inflammation is one of the characteristics of CRC. In the microenvironment of CRC, dense infiltrate of immune cells stimulates the secretion of proinflammatory cytokines, such as interleukin- (IL-) 6, IL-8, IL$1 \beta$, and TNF- $\alpha$, which can synergistically activate signal transducer and activator of transcription 3 (STAT3), nuclear factor kappa-B (NF- $\kappa \mathrm{B})$, and hypoxia-inducible factor $1 \alpha$ (HIF-1 $\alpha$ ) pathways, resulting in progression of CRC $[44,45]$. As a well-known transcription factor and tumor suppresser, p53 protein drive cell apoptosis to avoid possibly cancer-inducing damaged DNA passing on to daughter cells. Not surprisingly, inactivation of the p53 pathway is often observed in CRC; binding of DNA to mutant p53 can amplify downstream protumor pathways [46].

Following, 15 compounds were recognized as the major bioactive compounds of the CHs in the treatment of CRC, with their importance in the herb-bioactive compoundtherapeutic target network (Figure 7 and Table 2). Most major bioactive compounds belong to the flavonoid family, including quercetin, wogonin, luteolin, kaempferol, 5,7,4' trihydroxy-8-methoxyflavone, formononetin, carthamidin, pinocembrin, and baicalein, all of which could be similarly used as chemopreventive agents, for their capacity of inducing cytotoxic apoptosis, anti-inflammation, antioxidation, and antiangiogenesis [47].

Quercetin was reported to induce cell cycle arrest and apoptosis, modulate estrogen receptors, alleviate oxidation, and inhibit angiogenesis in CRC [48]. Luteolin might suppress the proliferation and transformation of human CRC cells through antioxidation and miRNAs related pathways $[49,50]$. Kaempferol could stimulate apoptosis in CRC cells, in which p53 mediated caspases activation might play critical roles [51]. And kaempferol should have a synergistic effect with 5-fluorouracil (5-FU) in CRC cell lines through PI3K/Akt inactivation [52]. Wogonin was observed to stimulate apoptosis through facilitating cytoplasmic localization of p53 in vitro, and its effects of reducing tumor multiplicity and preserving colon length were verified in vivo [53]. Another study demonstrated wogonin might simultaneously induce autophagy, apoptosis, and cell cycle arrest via blocking PI3K/Akt and STAT3 pathways [54], which was similar to formononetin [55]. Moreover, pinocembrin could trigger Bax-dependent mitochondrial apoptosis in CRC cells [56, 57]. Pachymic acid was also reported to induce cell cycle arrest and apoptosis in many kinds of digestive cancers [58-61].

Eburicoic acid exerts satisfying anti-inflammatory activity of relieving inflammatory cytokine production, and suppression of $\mathrm{PI} 3 \mathrm{~K} / \mathrm{Akt} / \mathrm{mTOR} / \mathrm{NF}-\kappa \mathrm{B}$ pathway might be involved $[62,63]$. 5,7,4'-Trihydroxy-8-methoxyflavone might suppress inflammatory cytokine production both in vitro and in vivo [64]. Carthamidin might induce DNA damage and oxidative cell death in CRC cells [65]. Baicalein could repress CRC cells proliferation through lessening the ezrin gene's expression and increasing p53 pathway-related proteins [66], and it could suppress invasion of CRC cells by blocking extracellular regulated protein kinases (ERK) pathway [67].

Next, VEGFA, CASP3, MYC, CYP1Y1, and NFKB1 were identified as key targets of the $\mathrm{CHs}$ in the treatment of CRC, with their contributions in the PPI network (Figure 4) and prognostic significance in CRC patients (Figure 5). Angiogenesis is responsible for tumor vascularization degree, growth, and metastasis. VEGFA is acknowledged as a significant proangiogenic factor, which is frequently overexpressed in metastatic CRC patients [68]. Besides, miRNAs, such as miR-203, miR-497, and miR-26a, might resist CRC by targeting VEGFA $[69,70]$. CASP3 activation triggers anticancer apoptosis and could be used as a marker of 


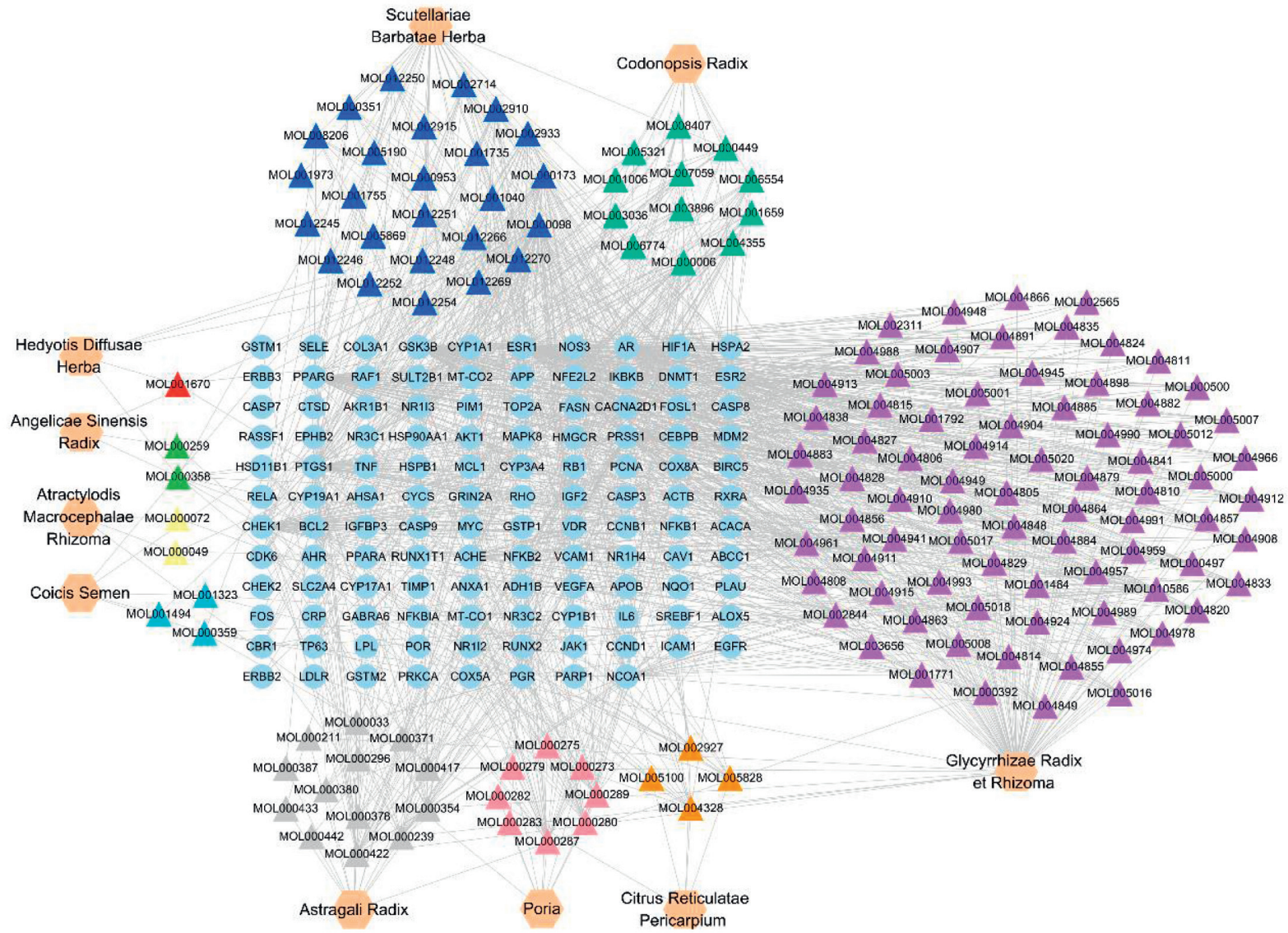

Figure 7: The herb-bioactive compound-therapeutic target network of the $\mathrm{CHs}$. The hexagonal nodes represent the $\mathrm{CHs}$, the triangle nodes represent the bioactive compounds, the circular nodes represent the therapeutic targets, and the edges represent the interactions among them.

apoptosis-targeted treatment response [71]. MYC is a family of transcriptional regulators, containing $\mathrm{c}-\mathrm{Myc}$, $\mathrm{n}-\mathrm{Myc}$, and $\mathrm{l}-\mathrm{Myc}$ in mammals. Deregulation of c-Myc exists in $70 \%$ of CRC, which could partly explain genome instability, tumorigenesis, and other malignant behaviors of CRC cells [72, 73]. CYP1A1 is used to be infamous for producing severe carcinogens. Although still controversial, CYP1A1 had been proposed more importance in detoxification, which might provide protective effects against some oral carcinogens in CRC patients [74]. NFKB1 belongs to NF- $\kappa \mathrm{B}$ family, which consists of a class of transcription factors working as central regulators of inflammatory pathways, cell proliferation, and apoptosis [75]. Activation of NF- $\kappa \mathrm{B}$ had been frequently observed in CRC patients, associated with worse outcomes [76]. Based on the above discussions, we could summarize VEGFA as an unfavorable factor, while CASP3, MYC, and CYP1Y1 as favorable factors in CRC, which was consistent with the results of survival analyses. In the contrary, our survival analysis showed that NFKB1 was correlated with longer DFS in CRC patients; for this, further researches are still needed.
In the end, the molecular docking simulation (Table 3 and Figure 6) showed a great majority of the 15 major bioactive compounds might bind firmly to VEGFA, CASP3, MYC, and especially CYP1Y1 proteins and thus afforded the possibility for them to exert their pharmacological activities. The molecular docking simulation strengthened all the above investigations to some extent, but experimental validations are still needed.

\section{Conclusion}

In summary, this study combined data mining and network pharmacology approach to identify ten the $\mathrm{CHs}$, fifteen major bioactive compounds (quercetin, wogonin, luteolin, etc.), and five key therapeutic targets (VEGF, CASP3, MYC, CYP1Y1, and NFKB1) in the treatment of CRC. Additionally, the underlying mechanisms of action of the $\mathrm{CHs}$ might be apoptosis induction, transcription modulation, and inflammation suppression, and microRNAs, TNF, apoptosis, PI3K-Akt, and p53 signal pathways are participated. This study threw light on the anti-CRC mechanisms of the $\mathrm{CHs}$ in a holistic manner, which might provide novel insights for 
therapeutic strategies and further studies. However, further experiments are still necessary.

\section{Data Availability}

The information of the clinical studies included in this study is provided in Supplementary Table 1. All data that support the findings of this study are publicly available from the databases mentioned in the Materials and Methods section.

\section{Conflicts of Interest}

The authors declare that there are no conflicts of interest regarding the publication of this paper.

\section{Supplementary Materials}

A list of the clinical studies included in this study is provided in Supplementary Table 1. (Supplementary Materials)

\section{References}

[1] F. Bray, J. Ferlay, I. Soerjomataram, R. L. Seigal, L. A. Torre, and A. Jemal, "Global cancer statistics 2018: GLOBOCAN estimates of incidence and mortality worldwide for 36 cancers in 185 countries," CA: A Cancer Journal for Clinicians, vol. 68, no. 6, pp. 394-424, 2018.

[2] N. Siegel and E. Giovannucci, "Global burden of colorectal cancer: emerging trends, risk factors and prevention strategies," Nature Reviews Gastroenterology \& Hepatology, vol. 16, no. 12, pp. 713-732, 2019.

[3] E. Dekker, P. J. Tanis, J. L. A. Vleugels, P. M. Kasi, and M. B. Wallace, "Colorectal cancer," The Lancet, vol. 394, no. 10207, pp. 1467-1480, 2019.

[4] C.-Y. Wang, H.-Z. Ding, X. Tang, and Z.-G. Li, "Comparative analysis of immune function, hemorheological alterations and prognosis in colorectal cancer patients with different traditional Chinese medicine syndromes," Cancer Biomarkers, vol. 21, no. 3, pp. 701-710, 2018.

[5] F. M. Li, K. Cresswell, A. S. Myint, and A. G. Renehan, "Is "watch-and-wait" after chemoradiotherapy safe in patients with rectal cancer?," BMJ (Clinical Research ed.), vol. 363, Article ID k4472, 2018.

[6] D. Papamichael and R. Glynne-Jones, "Identifying patients who may benefit from oxaliplatin-containing perioperative chemo (radio) therapy for rectal cancer," Annals of Oncology, vol. 29, no. 8, pp. 1616-1618, 2018.

[7] D. S. Keller, M. Berho, R. O. Perez, S. D. Wexner, and M. Chand, "The multidisciplinary management of rectal cancer," Nature Reviews Gastroenterology \& Hepatology, vol. 17, no. 7, p. 414, 2020.

[8] C. Perez and R. Glynne-Jones, "Challenges behind proving efficacy of adjuvant chemotherapy after preoperative chemoradiation for rectal cancer," The Lancet Oncology, vol. 18, no. 6, pp. e354-e363, 2017.

[9] F. Qi, L. Zhao, A. Zhou et al., "The advantages of using traditional Chinese medicine as an adjunctive therapy in the whole course of cancer treatment instead of only terminal stage of cancer," Bioscience Trends, vol. 9, no. 1, pp. 16-34, 2015.

[10] Y. Zhang, Y. Liang, and C. He, "Anticancer activities and mechanisms of heat-clearing and detoxicating traditional chinese herbal medicine," Chinese Medicine, vol. 12, p. 20, 2017.

[11] S. I. Lee, J. A. Bae, Y. S. Ko, H. Kim et al., "Geijigajakyak decoction inhibits the motility and tumorigenesis of colorectal cancer cells," BMC Complementary and Alternative Medicine, vol. 16, no. 1, p. 288, 2016.

[12] X. Lin, Z. Yi, J. Diao et al., "ShaoYao decoction ameliorates colitis-associated colorectal cancer by downregulating proinflammatory cytokines and promoting epithelial-mesenchymal transition," Journal of Translational Medicine, vol. 12, no. 1, p. 105, 2014.

[13] X. Shao, S. M. Saud, F. Wang et al., "Protective effect of ShaoYao decoction on colitis-associated colorectal cancer by inducing Nrf2 signaling pathway," Journal of Ethnopharmacology, vol. 252, p. 112600, 2020.

[14] Y. He, P. Liu, Y. Fang et al., "The effect of long-term traditional Chinese medicine treatment on survival time of colorectal cancer based on propensity score matching: a retrospective cohort study," Evidence-Based Complementary and Alternative Medicine: Ecam, vol. 2020, Article ID 7023420, 12 pages, 2020.

[15] Y.-T. Kuo, T.-T. Chang, C.-H. Muo et al., "Use of complementary traditional Chinese medicines by adult cancer patients in Taiwan: a nationwide population-based study," Integrative Cancer Therapies, vol. 17, no. 2, pp. 531-541, 2018.

[16] L. Zhang, L. Bi, Y. Lu et al., "Cancer chemoprevention and therapy using Chinese herbal medicine," Biological Procedures Online, vol. 20, p. 1, 2018.

[17] S. Zhang, L. Shi, D. Mao et al., "Use of jianpi jiedu herbs in patients with advanced colorectal cancer: a systematic review and meta-analysis," Evidence-Based Complementary and Alternative Medicine: Ecam, vol. 2018, Article ID 6180810, 13 pages, 2018.

[18] S.-S. Liu, Q. Shi, H.-J. Li et al., "Right- and left-sided colorectal cancers respond differently to traditional Chinese medicine," World Journal of Gastroenterology, vol. 23, no. 42, pp. 76187625, 2017.

[19] X.-J. Yang, X.-X. Song, L.-B. Wei, and Z.-G. Wang, "Study of the distribution patterns of the constituent herbs in classical Chinese medicine prescriptions treating respiratory disease by data mining methods," Chinese Journal of Integrative Medicine, vol. 19, no. 8, pp. 621-628, 2013.

[20] M. Wang, Z. Zhou, X. Wang et al., "Network pharmacologyguided development of a novel integrative regimen to prevent acute graft-vs.-host disease," Frontiers in Pharmacology, vol. 9, p. $1440,2018$.

[21] P. Poornima, J. D. Kumar, Q. Zhao, M. Blunder, and T. Efferth, "Network pharmacology of cancer: from understanding of complex interactomes to the design of multitarget specific therapeutics from nature," Pharmacological Research, vol. 111, pp. 290-302, 2016.

[22] L. Blunder and G. Rastelli, "Molecular docking: shifting paradigms in drug discovery," International Journal of Molecular Sciences, vol. 20, no. 18, 2019.

[23] J. Ru, P. Li, J. Wang et al., "TCMSP: a database of systems pharmacology for drug discovery from herbal medicines," Journal of Cheminformatics, vol. 6, p. 13, 2014.

[24] H.-Y. Xu, Y.-Q. Zhang, Z.-M. Liu et al., "ETCM: an encyclopaedia of traditional Chinese medicine," Nucleic Acids Research, vol. 47, no. D1, pp. D976-D982, 2019.

[25] Z. Chen, F. Guo, Y. Wang et al., "BATMAN-TCM: a bioinformatics analysis tool for molecular mechanism of traditional Chinese medicine," Scientific Reports, vol. 6, Article ID 21146, 2016. 
[26] B. Li, J. Rui, X. Ding, Y. Chen, and X. Yang, "Deciphering the multicomponent synergy mechanisms of SiNiSan prescription on irritable bowel syndrome using a bioinformatics/ network topology based strategy," Phytomedicine, vol. 63, Article ID 152982, 2019.

[27] N. Rappaport, S. Fishilevich, R. Nudel et al., "Rational confederation of genes and diseases: NGS interpretation via GeneCards, MalaCards and VarElect," Biomedical Engineering Online, vol. 16, no. 1, p. 72, 2017.

[28] J. Piñero, J. M. Ramírez-Anguita, J. Saüch-Pitarch et al., "The DisGeNET knowledge platform for disease genomics: 2019 update," Nucleic Acids Research, vol. 45, no. D1, pp. D833-D839, 2019.

[29] A. P. Davis, C. J. Grondin, R. J. Johnson et al., "The comparative toxicogenomics database: update 2019," Nucleic Acids Research, vol. 39, pp. D1067-D1072, 2019.

[30] G. Dennis, B. T. Sherman, D. A. Hosack et al., "DAVID: database for annotation, visualization, and integrated discovery," Genome Biology, vol. 4, no. 5, P3 pages, 2003.

[31] D. Szklarczyk, J. H. Morris, H. Cook et al., "The STRING database in 2017: quality-controlled protein-protein association networks, made broadly accessible," Nucleic Acids Research, vol. 45, no. D1, pp. D362-D368, 2017.

[32] P. Shannon, A. Markiel, O. Ozier et al., "Cytoscape: a software environment for integrated models of biomolecular interaction networks," Genome Research, vol. 13, no. 11, pp. 2498-2504, 2003.

[33] Q. Zeng, L. Li, W. Siu et al., “A combined molecular biology and network pharmacology approach to investigate the multitarget mechanisms of Chaihu Shugan San on Alzheimer's disease," Biomedicine \& Pharmacotherapy, vol. 120, p. 109370, 2019.

[34] H. Jin, G. Zhang, L. Zhang et al., "Comprehensive review of web servers and bioinformatics tools for cancer prognosis analysis," Frontiers in Oncology, vol. 10, p. 68, 2020.

[35] J.-M. Yang and C.-C. Chen, "GEMDOCK: a generic evolutionary method for molecular docking," Proteins: Structure, Function, and Bioinformatics, vol. 55, no. 2, pp. 288-304, 2004.

[36] H. M. Berman, J. D. Westbrook, Z. Feng et al., "The protein data bank," Nucleic Acids Research, vol. 28, no. 1, pp. 235-242, 2000.

[37] T. Sterling and J. J. Irwin, "Zinc 15-ligand discovery for everyone," Journal of Chemical Information and Modeling, vol. 55, no. 11, pp. 2324-2337, 2015.

[38] W. Taixiang, A. J. Munro, and L. Guanjian, "Chinese medical herbs for chemotherapy side effects in colorectal cancer patients," The Cochrane Database of Systematic Reviews, vol. 25, no. 1, Article ID D4540, 2005.

[39] M. Chen, B. H. May, I. W. Zhou, D. M.-Y. Sze, C. C. Xue, and A. L. Zhang, "Oxaliplatin-based chemotherapy combined with traditional medicines for neutropenia in colorectal cancer: a meta-analysis of the contributions of specific plants," Critical Reviews in Oncology/Hematology, vol. 105, pp. 18-34, 2016.

[40] M. H. Sze, B. H. May, I. W. Zhou, A. L. Zhang, and C. C. Xue, "Integrative medicine for relief of nausea and vomiting in the treatment of colorectal cancer using oxaliplatin-based chemotherapy: a systematic review and meta-analysis," Phytotherapy Research, vol. 30, no. 5, pp. 741-753, 2016.

[41] M. Zhang, B. H. May, I. W. Zhou, C. C. L. Xue, and A. L. Zhang, "FOLFOX 4 combined with herbal medicine for advanced colorectal cancer: a systematic review," Phytotherapy Research, vol. 28, no. 7, pp. 976-991, 2014.
[42] S. Xue, A. Mokhtarzadeh, E. Baghbani et al., "The role of microRNAs involved in PI3-kinase signaling pathway in colorectal cancer," Journal of Cellular Physiology, vol. 234, no. 5, pp. 5664-5673, 2019.

[43] J. Asadi, F. Ke, T. Chen et al., "MicroRNAs that regulate PTEN as potential biomarkers in colorectal cancer: a systematic review," Journal of Cancer Research and Clinical Oncology, vol. 146, no. 4, pp. 809-820, 2020.

[44] V. D. Simone, E. Franzè, G. Ronchetti et al., "Th17-type cytokines, IL- 6 and TNF- $\alpha$ synergistically activate $\mathrm{STAT}_{3}$ and NF-kB to promote colorectal cancer cell growth," Oncogene, vol. 34, no. 27, pp. 3493-3503, 2015.

[45] J. Hallajzadeh, P. Maleki Dana, M. Mobini et al., "Targeting of oncogenic signaling pathways by berberine for treatment of colorectal cancer," Medical Oncology (Northwood, London, England), vol. 37, no. 6, p. 49, 2020.

[46] A. A. Farooqi, M. De La Roche, M. B. A. Djamgoz, and Z. H. Siddik, "Overview of the oncogenic signaling pathways in colorectal cancer: mechanistic insights," Seminars in Cancer Biology, vol. 58, pp. 65-79, 2019.

[47] T. Siddik, O. C. Vlad, L. C. Miclea et al., "Alleviation of multidrug resistance by flavonoid and non-flavonoid compounds in breast, lung, colorectal and prostate cancer," International Journal of Molecular Sciences, vol. 21, no. 2, p. 401, 2020.

[48] D. Saber G, K. Mojtaba, Y. Bahman et al., "Quercetin: a functional dietary flavonoid with potential chemo-preventive properties in colorectal cancer," Journal of Cellular Physiology, vol. 233, no. 9, pp. 6544-6560, 2018.

[49] Q. Zuo, R. Wu, X. Xiao et al., "The dietary flavone luteolin epigenetically activates the Nrf2 pathway and blocks cell transformation in human colorectal cancer HCT116 cells," Journal of Cellular Biochemistry, vol. 119, no. 11, pp. 95739582, 2018.

[50] Y. Yao, C. Rao, G. Zheng, and S. Wang, "Luteolin suppresses colorectal cancer cell metastasis via regulation of the miR-384/ pleiotrophin axis," Oncology Reports, vol. 42, no. 1, pp. 131-141, 2019.

[51] J.-B. Choi, J.-H. Kim, H. Lee et al., "Reactive oxygen species and p53 mediated activation of p38 and Caspases is critically involved in kaempferol induced apoptosis in colorectal cancer cells," Journal of Agricultural and Food Chemistry, vol. 66, no. 38, pp. 9960-9967, 2018.

[52] Q. Li, L. Wei, S. Lin et al., "Synergistic effect of kaempferol and 5-fluorouracil on the growth of colorectal cancer cells by regulating the PI3K/Akt signaling pathway," Molecular Medicine Reports, vol. 20, no. 1, pp. 728-734, 2019.

[53] Q. Feng, H. Wang, J. Pang et al., "Prevention of wogonin on colorectal cancer tumorigenesis by regulating p53 nuclear translocation," Frontiers in Pharmacology, vol. 9, p. 1356, 2018.

[54] H. Tan, X. Li, W. H. Yang, and Y. Kang, “A flavone, wogonin from scutellaria baicalensis inhibits the proliferation of human colorectal cancer cells by inducing of autophagy, apoptosis and G2/M cell cycle arrest via modulating the PI3K/Akt and STAT3 signalling pathways," Journal of B.U.ON.: Official Journal of the Balkan Union of Oncology, vol. 24, no. 3, pp. 1143-1149, 2019.

[55] S. K. L. Ong, M. K. Shanmugam, L. Fan et al., "Focus on formononetin: anticancer potential and molecular targets," Cancers, vol. 11, no. 5, 2019.

[56] M. A. S. Kumar, M. Nair, P. S. Hema, J. Mohan, and T. R. Santhoshkumar, "Pinocembrin triggers Bax-dependent 
mitochondrial apoptosis in colon cancer cells," Molecular Carcinogenesis, vol. 46, no. 3, pp. 231-241, 2007.

[57] N. Mohan, S. Biswas, A. Hosur Shrungeswara et al., "Pinocembrin enriched fraction of Elytranthe parasitica (L.) Danser induces apoptosis in HCT 116 colorectal cancer cells," Journal of Infection and Chemotherapy, vol. 23, no. 6, pp. 354-359, 2017.

[58] C. Basu Mallik, J. Ma, and D. Cai, "Pachymic acid inhibits the tumorigenicity of gastric cancer cells by the mitochondrial pathway," Anti-cancer Drugs, vol. 28, no. 2, pp. 170-179, 2017.

[59] S. Cheng, K. Swanson, I. Eliaz et al., "Pachymic acid inhibits growth and induces apoptosis of pancreatic cancer in vitro and in vivo by targeting ER stress," PLoS One, vol. 10, no. 4, Article ID e122270, 2015.

[60] K. X. Sun and H. W. Xia, "Pachymic acid inhibits growth and induces cell cycle arrest and apoptosis in gastric cancer SGC7901 cells," Oncology Letters, vol. 16, no. 2, pp. 2517-2524, 2018.

[61] Y. Chen, P. Lian, Y. Liu, and K Xu, "Pachymic acid inhibits tumorigenesis in gallbladder carcinoma cells," International Journal of Clinical and Experimental Medicine, vol. 8, no. 10, pp. 17781-17788, 2015.

[62] J. Xu, P. Zhang, H. He et al., "Eburicoic acid from Laetiporus sulphureus (Bull.:Fr.) Murrill attenuates inflammatory responses through inhibiting LPS-induced activation of PI3K/ Akt/mTOR/NF- $\kappa$ B pathways in RAW264.7 cells," NaunynSchmiedeberg's Archives of Pharmacology, vol. 390, no. 8, pp. 845-856, 2017.

[63] J.-S. Se, S.-S. Huang, T.-H. Lin et al., "Analgesic and antiinflammatory bioactivities of eburicoic acid and dehydroeburicoic acid isolated from Antrodia camphorata on the inflammatory mediator expression in mice," Journal of Agricultural and Food Chemistry, vol. 61, no. 21, pp. 5064-5071, 2013.

[64] P. J. Lee, W. C. Huang, M. C. Hsieh et al., "Flavones isolated from Scutellariae radix suppress propionibacterium acnesinduced cytokine production in vitro and in vivo," Molecules (Basel, Switzerland), vol. 21, no. 1, p. E15, 2015.

[65] V. Chen, R. E. Staub, S. Baggett et al., "Identification and analysis of the active phytochemicals from the anti-cancer botanical extract Bezielle," PLoS One, vol. 7, no. 1, Article ID e30107, 2012.

[66] Z. Chen, R. Hou, S. Gao, D. Song, and Y. Feng, "Baicalein inhibits proliferation activity of human colorectal cancer cells HCT116 through downregulation of ezrin," Cellular Physiology and Biochemistry, vol. 49, no. 5, pp. 2035-2046, 2018.

[67] Y. Song, J. Xu, and B. Yan, "The anti-metastatic effect of baicalein on colorectal cancer," Oncology Reports, vol. 37, no. 4, pp. 2317-2323, 2017.

[68] M. Canavese, D. T. M. Ngo, G. J. Maddern, J. E. Hardingham, T. J. Price, and E. Hauben, "Biology and therapeutic implications of VEGF-A splice isoforms and single-nucleotide polymorphisms in colorectal cancer," International Journal of Cancer, vol. 140, no. 10, pp. 2183-2191, 2017.

[69] C. Hardingham, Z. Huang, X. Mo et al., "The circular RNA 001971/miR-29c-3p axis modulates colorectal cancer growth, metastasis, and angiogenesis through VEGFA," Journal of Experimental \& Clinical Cancer Research, vol. 39, no. 1, p. 91, 2020.

[70] W. Zhang, C. Zou, L. Pan et al., "MicroRNA-140-5p inhibits the progression of colorectal cancer by targeting VEGFA," Cellular Physiology and Biochemistry, vol. 37, no. 3, pp. 1123-1133, 2015.
[71] D. L. Xu, J. T. Engle, E. A. Griffin et al., "Imaging caspase-3 activation as a marker of apoptosis-targeted treatment response in cancer," Molecular Imaging and Biology, vol. 17, no. 3, pp. 384-393, 2015.

[72] M. Miller, T. Usui, H. Yamawaki, and K. Sasaki, "Emerging roles of C-Myc in cancer stem cell-related signaling and resistance to cancer chemotherapy: a potential therapeutic target against colorectal cancer," International Journal of Molecular Sciences, vol. 20, no. 9, 2019.

[73] L. Brandl, N. Kirstein, J. Neumann et al., "The c-MYC/ NAMPT/SIRT1 feedback loop is activated in early classical and serrated route colorectal cancer and represents a therapeutic target," Medical Oncology (Northwood, London, England), vol. 36, no. 1, 2018.

[74] M. Mescher and T. Haarmann-Stemmann, "Modulation of CYP1A1 metabolism: from adverse health effects to chemoprevention and therapeutic options," Pharmacology \& Therapeutics, vol. 187, pp. 71-87, 2018.

[75] K. Taniguchi and M. Karin, "NF- $\kappa$ B, inflammation, immunity and cancer: coming of age," Nature Reviews Immunology, vol. 18, no. 5, pp. 309-324, 2018.

[76] C. Porta, A. Ippolito, F. M. Consonni et al., "Protumor steering of cancer inflammation by p50 NF- $\kappa \mathrm{B}$ enhances colorectal cancer progression," Cancer Immunology Research, vol. 6, no. 5, pp. 578-593, 2018. 